\title{
La cohérence des textes : entre préoccupations didactiques et questionnements linguistiques
}

\author{
Fabienne Rondelli \\ Modèles, Dynamiques, Corpus (MoDyCo), Nanterre \\ fabienne.rondelli@wanadoo.fr
}

\section{Introduction}

Depuis la parution de la brochure ministérielle La maîtrise de la langue à l'école en 1992, qui annonçait le double objectif de faire le point sur les travaux des chercheurs dans le domaine de la langue et de fournir aux enseignants des pistes pédagogiques susceptibles de les aider à intégrer les acquis de ces recherches dans leurs pratiques, la cohérence dans ses dimensions pragmatique, sémantique, structurelle est devenue à première vue une notion de base du travail sur le texte. Les derniers programmes pour l'école, réécrits en 2007, précisent à leur tour qu' " en situation d'écriture, il convient de rendre les élèves sensibles aux ruptures qui surviennent souvent dans leurs productions et de les conduire à rétablir la cohérence du texte qu'ils produisent $»^{2}$. Cette prescription implique 1) que les enseignants doivent être capables de juger de la cohérence d'un texte 2) qu'ils puissent en dégager pour les élèves d'éventuels besoins langagiers 3) qu'ils sachent mettre en place des dispositifs d'aide à l'élaboration de la cohérence. Ainsi, la cohérence apparaît comme un objet incontournable dans le cadre de l'enseignement / apprentissage du français. Les professeurs des écoles, pour peu qu'on les interroge, montrent d'ailleurs qu'ils en possèdent une connaissance déclarative, mais leur conception exprimée se réduit assez souvent aux aspects formels de quelques faits de cohésion ${ }^{3}$ (chaînes anaphoriques, connecteurs, temps verbaux...) qui sont ceux que l'on retrouve par ailleurs dans le chapitre grammaire des programmes officiels.

Il serait sans doute nécessaire d'analyser les transpositions des analyses théoriques de la cohérence en formation et les moyens d'enseignement effectifs des enseignants pour mieux comprendre cette réduction assez générale de la critérisation déclarée de la cohérence aux aspects structurels. Pour notre part, dans le cadre de cet article, nous choisissons un axe complémentaire nous permettant d'aller plus loin dans la prise en compte des représentations de la cohérence des enseignants. En effet, nous pensons que les professeurs des écoles s'appuient généralement sur des critères de cohérence bien plus larges, bien plus complexes que les critères déclarés.

Si nous admettons cette hypothèse, plusieurs questions s'imposent :

- Quels sont précisément les critères sur lesquels les professeurs des écoles s'appuient pour juger la cohérence d'un texte d'élève?

- En quoi ces critères ont-ils à voir avec les définitions de la cohérence proposées par les chercheurs ?

- En quoi la confrontation de ces deux réalités peut être utile à la recherche en didactique ?

L'objectif général de cet article est de proposer des éléments de réponse aux deux premières questions, car nos préoccupations didactiques concernant la cohérence impliquent de façon incontournable un questionnement linguistique.

La cohérence, sous une apparente unité ${ }^{4}$, est un objet théorique multiforme, dont s'emparent nombre de champs de recherche en linguistique, proposant des concepts et des méthodes peu homogènes. Par ailleurs, réfléchir à la question de la cohérence des textes à l'école implique, comme nous l'avons évoqué, de se préoccuper des représentations qu'ont les enseignants à propos de cette notion. Aussi, toute réflexion sur une possible didactique de la cohérence doit, selon nous, s'appuyer sur ces deux piliers : l'apport théorique des spécialistes et les «proto-théories » proposées par les professeurs des écoles.

\section{Le cadre théorique et méthodologique}

\subsection{A la recherche des définitions de la cohérence}


La plupart des études concernant la cohérence en proposent d'emblée une définition en fonction du paradigme théorique de référence. L'enjeu de cette recherche est différent : nous confrontons des critères définitionnels avancés par des théories du texte aux critères implicites, spontanés des professeurs des écoles. Il ne s'agit cependant pas de convoquer, dans une perspective synchronique ou diachronique, toutes les analyses de la notion, mais de construire un cadre théorique constitué de concepts opératoires issus de champs théoriques ciblés qui seront autant de filtres successifs appliqués aux mêmes données.

Pour cerner la notion de cohérence, il parait raisonnable de partir d'un principe très puissant : le principe de coopération de Grice (1979) : soit plusieurs énoncés engendrés à la suite, le récepteur considère qu'il doit exister une façon de lier ces énoncés, produits dans une certaine continuité pour montrer qu'ils entretiennent un certain rapport de sens. Partant, le sujet s'efforce de construire toutes les inférences nécessaires pour trouver le lien sémantique entre les énoncés. Cependant, deux phénomènes semblent rendre les choses un peu plus compliquées : d'une part, certains textes sont jugés non cohérents par leur destinataire : il est démontré en effet que le récepteur s'arrête prioritairement à des associations de sens commun et ne fait pas spontanément d'inférences qui ne soient pas a minima prévisibles (Sperber et Wilson, 1989). D'autre part, un texte ne peut être traité en une seule fois, en réception ou en production (Charolles, 1995). C'est pourquoi les scripteurs intègrent généralement des traits internes au texte luimême qui sont constitutifs de sa cohérence et aident à la fois à la production et à la compréhension : des marques linguistiques contribuent à l'organisation du texte en un tout signifiant.

Au final, comment s'élabore la cohérence?

On retiendra deux principes : 1) un texte est constitué «d'unités » liées 2) la cohérence semble relever à la fois de l'intelligibilité (logique du texte) et du rapport personnel au texte (expériences du sujet).

Ces deux axes majeurs, considérés séparément ou en interaction, sont présents dans toutes les propositions scientifiques de définition de la cohérence. Ils sont singulièrement discutés par trois champs de recherche plutôt récents ${ }^{5}$ : l'analyse textuelle des discours; la sémantique textuelle; la psycholinguistique textuelle. Ces trois courants nous semblent particulièrement intéressants à confronter dans la perspective de la compréhension des jugements de cohérence des enseignants, car ils posent la difficile question de la tension entre cohérence comme fait de texte et cohérence comme fait du sujet.

\subsubsection{Cohérence et régularité intertextuelle}

L'analyse textuelle des discours décrit les régularités d'agencement d'un texte qui lui confèrent la propriété de former une unité cohésive et cohérente. Jean-Michel Adam est l'un des linguistes du texte qui, pour atteindre cet objectif, propose une vision systémique du texte et de ses entours. Dans cette perspective, il rend opératoire le rapprochement récent entre linguistique textuelle et analyse de discours en montrant que « la cohérence est une notion au croisement de la linguistique textuelle et de l'analyse de discours » (Adam, $2002: 100$ ). S'il rejoint Charolles (2005) sur le fait que «le jugement de textualité et de cohérence globale est, en grande partie, fondé sur l'établissement, par l'interprétant, d'une signification d'ensemble» (Adam, 1999: 31.), il ajoute cependant un point crucial : «à condition toutefois de mettre cette intentionnalité globale en rapport avec la dimension sociodiscursive de toute action langagière $»$. (Adam, $1999: 31$.)

Cet appui sur le concept d' « action langagière », qui fait partie de l'appareil notionnel du psychologue interactioniste Jean-Paul Bronckart (1997), invite à considérer le langage comme une production interactive associée aux activités sociales, production qui implique la constitution de représentations. Les représentations collectives progressivement constituées mais toujours en évolution permettent l'intercompréhension entre des communautés verbales. Ces pôles d'intercompréhension sont qualifiés par Bronckart de formations socio -langagières et débouchent sur les divers genres de textes (1997). Le sujet va mobiliser ses représentations en lien avec les conditions d'énonciation dans lesquelles il se trouve. Ce sera sa «base d'orientation » ${ }^{6}$ pour l'élaboration - compréhension d'un texte. Les textes sont donc des produits verbaux résultant de la tension entre une situation d'énonciation singulière et les régularités intertextuelles d'un genre. Ces régularités s'actualisent nécessairement dans le cadre des ressources 
linguistiques offertes par une langue naturelle donnée : les mondes construits par le sujet, qu'ils soient fictionnels ou ancrés dans le réel, ne sont attestables qu'à partir des formes linguistiques qui les sémiotisent, et ils sont donc solidaires de ces formes linguistiques. Il existe de ce fait une architecture interne des textes : un texte est constitué d'unités clairement identifiables qui s'articulent entre elles, qui se lient entre elles. Pour Jean-Michel Adam, un texte est constitué de cinq types de liage : les connexions (connecteurs et organisateurs textuels), les implicitations (ellipses, présupposés, sous-entendus), les chaînes d'actes de discours (les actes de discours dans un texte sont liés et hiérarchisés), les liages du signifiant (répétitions de phonèmes, syllabes, lexèmes, groupes morpho-syntaxiques), les liages du signifié (anaphores, isotopies) (Adam, 2005). Pour lui, le texte existe si ces types de lien sont effectivement actualisés : «chacune de ces cinq opérations est un facteur de textualité, mais aucune ne suffit seule à faire d'un texte une unité cohérente ». (Adam 2005, 85). Il est cependant également nécessaire que ces opérations s'organisent en configurations identifiables (donc en formations sociolangagières) : les séquences textuelles. Elles sont plus ou moins typées et ont comme caractéristiques le fait de correspondre à des catégories de relations macro-sémantiques mémorisées par imprégnation socioculturelle. Le texte est donc un matériau verbal construit dans le cadre d'une formation langagière le référant à un genre et dont l'organisation interne, en appui sur des unités discrètes et localisables, en dénote la cohérence.

\subsubsection{Cohérence et parcours interprétatifs}

Les théories interprétatives (sémantique interprétative, sémiotique textuelle) cherchent à mettre au jour la manière dont les mots, parce qu'ils sont inscrits dans un cotexte, provoquent des effets de sens. Nous nous intéressons particulièrement à l'idée de parcours interprétatif.

Lorsque François Rastier analyse la cohérence comme l'« unité d'une séquence linguistique, définie par ses relations avec son entour » (Rastier 1987, 273), il confirme l'idée qu'un texte résulte d'une tension entre des caractéristiques intrinsèques et des caractéristiques extrinsèques. Il est d'accord sur le fait que le texte est un ensemble constitué : « entre la forme matérielle du texte et ses interprétations, tout un système de normes, dont témoignent les structures textuelles, assurent la médiation indispensable » (Rastier, 2001, 23). Cependant, il développe une théorie sémantique qui réfute l'idée d'organisation hiérarchisée d'unités clairement identifiées qui s'articuleraient par des opérations de liage. Il remet particulièrement en question la détermination, dans la construction du sens d'un texte, du local sur le global (Rastier, 2006). Au contraire, il propose de considérer la construction de la cohérence comme un parcours interprétatif, où le sens se construit dans une dynamique fondée sur une sélection thématique que réalise le récepteur à partir de scénarios intertextuels qu'il connaît. Autrement dit, chaque récepteur fait appel à sa compétence encyclopédique et intertextuelle pour interpréter un texte, en actualisant, au fur et à mesure de son avancée dans le texte, les propriétés sémantiques dont il a besoin (par exemple, dans le cadre encyclopédique du conte, l'occurrence loup activera des propriétés comme «parle», en revanche, dans un texte documentaire, le même lexème loup orientera le récepteur vers l'encyclopédie qui règle le monde «réel »). Le récepteur décide des propriétés qui doivent être activées ou narcotisées en faisant des hypothèses sur le topic ou les topics du texte (Eco, 1985). Déterminer le topic signifie avancer une hypothèse quant à une certaine régularité textuelle. Pour U. Eco, ce type de régularité est ce qui fixe « tant les limites que les conditions de cohérence d'un texte» (Eco, 1985: 114). La cohérence interprétative consiste en une redondance assurant une homogénéité sémantique. Le texte construit donc un effet de monde. Mais si les mots ouvrent des mondes, ils le font parfois sans suivre la linéarité du texte. Ainsi, il est fréquent dans la littérature que le début d'un texte suggère un topic pour finalement en proposer un autre. La cohérence textuelle se construit donc progressivement, dans un double mouvement d'anticipations et de traitement rétro-actifs et ne saurait se décrire en terme de lecture linéaire. On peut dire que si la représentation d'un monde est constituée par l'agencement de signifiés, elle dépasse la somme des signifiés : chaque phrase, isolée, peut s'entendre de différentes façons, mais c'est le texte dans sa globalité qui détermine la signification à privilégier dans chacune des phrases. De même, « la question du sens d'un mot [est] oiseuse tant qu'on ne sait pas où il est attesté...toute valeur est contextuelle » (Rastier, 2007). 


\subsubsection{Cohérence et intégration d'unités sémantiques}

Les cognitivistes et psycholinguistes expliquent le processus de construction - intégration sémantique en montrant par quelles opérations le sujet fait des liens de sens entre le connu, déjà lu et le nouveau, «en train d'être lu ». La réception textuelle est forcément une activité qui se déroule dans le temps, ce qui implique que l'on s'interroge sur les moyens cognitifs à la disposition des sujets pour activer non pas une signification stable et unique, mais une signification mouvante car en cours, une signification plus ou moins provisoire. Les psycholinguistes sont d'accord sur le fait qu'en cours de lecture, des connaissances en mémoire à long terme sont activées en réseau (Perruchet, 1988, Gombert, 1997), c'est-à-dire qu'à la lecture de chaque mot, de façon largement automatisée, les sens possibles d'un mot sont activés, puis très rapidement (quelques dixièmes de secondes), le sens conforme au contexte reste. Ainsi, à chaque stimulation linguistique, une signification renvoie à des significations associées. Or, cette élaboration " spontanée », automatisée est à la fois une aide et une entrave : une aide car l'accessibilité des unités appartenant au même réseau de connaissance qu'une première unité activée se trouve accrue: soit l'activation d'un scénario, tout le réseau de connaissances à disposition du sujet serait plus facilement disponible. Une entrave dans me sens où l'activation automatique implique la mise au premier plan d'unités non pertinentes. Intervient donc un second processus qui va exclure les éléments non pertinents. Cela veut dire que l'intégration sémantique se réalise en deux paliers : un palier horizontal et un palier vertical (Coirier et al., 1996). La composante horizontale consiste en la recherche par le sujet pour chaque information nouvelle d'une information ancienne à laquelle la rattacher. La composante verticale est plus contrôlée, car c'est celle qui conduit à l'élaboration d'une macrostructure, autrement dit, à une représentation du texte en fonction des connaissances du sujet et des scénarios mentaux à sa disposition. Ces « schémas » sont organisés en mémoire par association d'informations sur un objet, une situation, un évènement. Il est généralement précisé qu'ils ont deux fonctions essentielles :

- assurer la cohérence de la représentation du texte par la mise en lien des différents éléments.

- permettre l'anticipation et la prédiction d'éléments du texte.

Mais une autre fonction des schémas nous semble nécessaire à relever dans le cadre de notre étude : son rôle reconstructif (modèle de Spiro décrit par Coirier et al., 1996). Outre la mise en lien des informations antérieures avec les informations nouvelles, les schémas, selon Spiro 1980, peuvent être la source de reconstructions sémantiques : ainsi, s'il manque des éléments dans un texte, un sujet les intégrera parce qu'il met en lien le donné textuel avec ses propres scénarios. De même, si un texte propose des informations ne semblant pas congruentes, il est prouvé que les sujets font un rappel du texte en faisant intervenir leurs schémas et en réduisant les incohérences (Coirier et al., 1996). C'est le fait que le lecteur (dans notre cadre, l'évaluateur) peut donc commettre des erreurs reconstructives pour tenter de réduire le désaccord entre ce qu'il lit et sa propre représentation du monde proposé par le texte qui nous intéresse particulièrement.

Les trois approches théoriques convoquées montrent que la construction de la cohérence relève d'une tâche récurrente pour le lecteur : mettre en lien ce qui vient d'être lu avec ce qui arrive. Nous pensons que par l'analyse des jugements de cohérence des enseignants, nous pourrons repérer, de façon certes empirique et partielle, les appuis à la mise en lien des énoncés : les enseignants sont dans une posture particulière car ils sont à la fois lecteurs et correcteurs. Dans ce cadre, recherchent-ils à repérer plutôt le bon emploi de marques relatives à l'intelligibilité du texte (marques d'énonciation, texture microlinguistique) ou sont-ils lecteurs interprétants appréciant le dit et le non dit, les effets de fiction, le pouvoir d'intéressement des textes produits?

\subsection{Une démarche fondée sur la confrontation}

«Le texte est avant tout une pratique signifiante, rencontre d'un sujet et de sa langue. Le texte est aussi le théâtre même d'une production où se rejoignent le producteur du texte et son lecteur. » (Barthes, 1992 : 373.) 
Nous posons la question de la cohérence, non pas à partir de modèles préconstruits, mais en fonction des jugements spontanés de sujets dont le rapport à l'écriture et à la norme est spécifiquement construit : les enseignants. Pour ce faire, nous avons constitué un corpus composé des traces de l'évaluation de productions textuelles d'élèves de cycle 3 de l'école élémentaire. La situation socio-discursive des élèves est celle de l'exercice d'écriture ponctuel et non pas celle liée à un projet d'écriture tel que proposé dans les travaux majeurs en didactique du français ${ }^{7}$. La tâche d'écriture retenue relève donc des scénarios les plus classiques de l'école : écrire la suite d'un texte narratif ${ }^{8}$. Notons que cet exercice est régulièrement proposé par les évaluations nationales afin d'examiner les compétences scripturales des élèves. Une consigne complémentaire est donnée : insérer un dialogue. Nous ne détaillons pas les types de séances mis en place par les enseignants, notre but étant ici d'analyser les jugements. Nous donnons cependant ciaprès quelques justifications du choix du support et de la consigne :

Pourquoi un texte narratif ?

Le texte narratif pose en soi des problèmes spécifiques, car le récit est à la fois un texte référentiel et un texte fictionnel. Du point de vue de la cohérence, s'articulent la nécessité de respecter des règles du genre et la liberté de s'en détacher (principes complémentaires d'identité et de différence discutés dans le cadre des études sur le genre).

Pourquoi une suite de texte?

Le texte de départ ${ }^{9}$ ) nous semble tout particulièrement intéressant dans la perspective de l'analyse des jugements de cohérence car sa relative complexité peut induire un double effet :

$\rightarrow$ un effet facilitateur, dans le sens où il crée des images relevant de stéréotypes présents dans des textes patrimoniaux dont on peut faire l'hypothèse qu'ils font partie des savoirs culturels des élèves de cycle 3 (livresques, cinématographiques, vidéogrammiques) : le héros face au danger.

$\rightarrow$ un effet perturbateur, dans le sens où 1) il met en œuvre un discours du récit complexe tout particulièrement au niveau de l'ordre 2) il entremêle deux champs lexicaux opposés : le plaisir du bain / le danger de mort.

Pourquoi l'insertion d'un dialogue?

L'obligation d'insérer un dialogue (un polylogue en l'occurrence au vu du nombre de personnages qui peuvent prendre la parole) peut être source du même double effet :

$\rightarrow$ un effet facilitateur, tout particulièrement dans le cadre de l'univers représenté par le texte - source, car le discours rapporté contribue à un effet de mise en scène, de théâtralisation, il permet des marques d'émotion, des énoncés interrompus, il vise une dramatisation de l'échange.

$\rightarrow$ un effet perturbateur, car le changement constant de locuteur peut engendrer une discontinuité narrative. De plus, le polylogue pose des problèmes particuliers liés aux marques du discours rapporté, comme la gestion des tirets, qui manquants ou supplémentaires, risquent de décaler l'attribution des tours de parole entre les personnages.

Nous présentons dans le cadre de cet article une analyse des jugements de cinquante trois enseignants sur une même suite de texte, écrite par Précilia ${ }^{10}$. Ces enseignants correcteurs ont connaissance du contexte de recherche et corrigent des textes anonymés et dont l'orthographe a été rétablie. La consigne de correction est très générale : «Cette suite de texte vous parait-elle réussie ? Vous pouvez annoter le texte et ajouter un commentaire final». Le rétablissement de l'orthographe invite les enseignants à observer d'autres niveaux du produit écrit.

Nous commentons les jugements en fonction des trois filtres théoriques retenus mais selon des objectifs différents : en fonction de nos axes 1) et 2) soit les opérations linguistiques de continuité - discontinuité textuelles et la construction de la cohérence sémantique, nous confrontons les appuis des professeurs pour juger la cohérence du texte. Les outils que mettent à notre disposition les recherches de l'axe 3), soit la description des opérations mentales en jeu dans la mise en lien d'éléments textuels permettent de différencier les modes de lecture des textes des enseignants. Les axes sélectionnés autorisent une confrontation dans deux dimensions : une dimension inter-jugements où nous voyons les remarques et annotations de plusieurs enseignants sur un même objet textuel ; une dimension intra-jugement, où nous observons les choix et l'évolution d'un même correcteur dans son évaluation de la cohérence du texte de l'élève.

Ainsi, nous réalisons plusieurs lectures du même corpus. 


\section{$3 \quad$ Analyse des jugements}

Sur les cinquante trois enseignants, cinq ne proposent aucune annotation en cours de lecture. Le texte de l'élève reste vierge de toute trace. Tous les enseignants ont rédigé un commentaire final, parfois sous la forme d'une synthèse - reformulations des annotations-, parfois par l'intermédiaire d'analyses de faits textuels non évoqués dans les annotations. Les modalités énonciatives sont de deux sortes : soit une adresse directe à l'élève, soit un commentaire destiné au chercheur. Nous ne détaillons pas ici plus avant la teneur des actes illocutoires qui sont très divers dans leur intentionnalité et leur forme (conseil, injonction, jugement de valeur, questionnement, recommandation, etc.). Nous distinguons le signalement d'objets langagiers des commentaires afférents: en effet, le même fait peut être relevé pour être disqualifié ou au contraire validé comme une réussite. De manière identique, les commentaires relatifs à un même jugement peuvent être distincts, voire contradictoires.

\subsection{Effets de texte}

\subsubsection{Continuité thématique avec le texte amorce}

Les enseignants évaluent massivement l'enchaînement thématique avec le texte amorce du point de vue des personnages (29 mentions).

Disqualification du fait de l'absence de dénomination du « héros » Knut :

- prénoms nouveaux sans lien avec le texte initial $\rightarrow$ il n'est pas question du nageur Knut.

- le personnage principal du texte de départ n'apparaît pas.

- où est passé Knut ?

- les personnages sont changés (on ne parle plus de Knut)

- c'est Knut qui nageait !

Disqualification du fait de l'insertion de personnages :

- on ne sait pas qui sont ces personnages $($ Joël,...) $\rightarrow$ les compagnons ?

- on ne connaît pas les différents personnages (Joël, Jean-Laurent,...) L'élève aurait dû les présenter.

- l'élève ne décrit pas les nouveaux personnages.

- $\quad$ pourquoi ajouter ces personnages ?

- confusion entre les personnages : «ils atteignirent» a été compris comme plusieurs personnes, dont il n'était d'ailleurs pas question auparavant, et non comme le personnage principal et le requin.

Validation du fait du nombre de personnages intégrés :

- les personnages dénommés Joël, etc. par l'élève semblent être les 4 compagnons de Knut.

- le thème de l'histoire est repris puisque le requin est repris ainsi que les cinq compagnons.

- l'élève respecte le nombre de personnages présents dans le récit : 5 personnages.

Validation du fait du recours aux noms propres:

- l'élève a nommé les personnages, ce qui donne de la cohérence au texte.

- l'élève a nommé les personnages, ce qui lui a facilité la tâche pour en parler ensuite.

- Précilia nomme les personnages.

Ces remarques posent la question de l'actant se trouvant en position de thème. En effet, Précilia ne propose pas un enchaînement de type linéaire reprenant le pronom " ils » de la dernière phrase du texte amorce mais choisit une progression par un thème dérivé d'une information donnée dans le paratexte situé en amont du texte amorce. Knut et le requin n'occupent plus le premier plan, ce qui, pour certains professeurs des écoles, remet en question la cohérence thématique ; ce qui pour d'autres au contraire, permet de réintégrer un élément déjà donné : les compagnons deviennent des adjuvants. Le recours aux noms propres comme procédé de dénomination initiale est considéré comme une réussite dans la mesure où les actions de chacun des personnages vont pouvoir être spécifiées. Une annotation du type On ne sait 
pas qui sont ces personnages $($ Joël,...) $\rightarrow$ les compagnons ? relève dans ce cadre sans doute plus d'une demande d'expansion que d'une réelle disqualification : dans la tradition de l'écriture scolaire, on décrit a minima tout nouveau personnage ; il semble que les professeurs ayant disqualifié la liste initiale des prénoms considèrent que l'inférence menant aux cinq compagnons de Knut est trop lourde. Une remarque du type pourquoi ajouter ces personnages? demande en effet à l'élève de faire agir au premier plan les deux protagonistes : Knut et le requin. La lecture du texte paraît s'appuyer sur des indices de connexité interphrastique et non sur une progression thématique au niveau de l'ensemble du texte, ce que confirme l'annotation suivante qui interroge la compréhension par l'élève du texte amorce : confusion entre les personnages : "ils atteignirent » a été compris comme plusieurs personnes, dont il n'était d'ailleurs pas question auparavant, et non comme le personnage principal et le requin.

\subsubsection{Voix narrative}

Les remarques très nombreuses concernant les personnages ont sans doute aussi à voir avec la question de l'instance de narration ; un PE écrit d'ailleurs : les personnages rajoutés remplacent-ils le "nous »? Un autre entoure le pronom «nous » du texte amorce, les prénoms proposés par Précilia et précise : c'est un de ces personnages qui doit raconter l'histoire. Cependant, neuf enseignants seulement signalent comme problème de cohérence énonciative le passage d'un narrateur-personnage à un narrateur externe. La construction linguistique du texte amorce explique peut-être le peu de remarques : il se termine par deux phrases qui sont constituées d'une chaîne de désignateurs à la troisième personne : l'ombre, un nageur, qui, Knut, ils. Et aucun déictique n'est présent sur les trois dernières lignes.

\subsubsection{Discours rapporté}

39 enseignants commentent le discours rapporté, en faisant essentiellement des critiques formelles: certains estiment qu'il manque des guillemets, d'autres imposent les deux points après questions, d'autres encore sanctionnent la répétition du verbe dire; un PE remarque l'absence de la section de discours attributif après Mais bien sûr qui rétablirait une régularité sur l'ensemble du dialogue. Un correcteur attire l'attention sur la structure compositionnelle : il remarque que la partie discours est encadrée par deux blocs récits. Nous notons qu'aucun des enseignants correcteurs n'intervient sur le rôle du dialogue dans la logique du récit: le dialogue est dans le texte de Précilia le lieu de la résolution positive (après une résolution déceptive dans le premier bloc récit). Par ailleurs, aucun enseignant ne semble prêter attention à un choix énonciatif de l'élève qui est peut-être un embryon de rencontre entre le discours des personnages et le discours du narrateur : le point d'exclamation final. Qui l'assume? Le narrateur surpris de voir ses héros ne plus souhaiter sortir en mer ou les personnages guéris de toute velléité à l'exploit marin?

\subsubsection{Etapes de la séquence narrative prises en charge par l'élève}

La dimension structurelle donne lieu à des remarques de 13 enseignants.

Composition de la séquence narrative :

- l'histoire respecte l'irruption d'un évènement et de son déroulement, ce qui est très bien

- il produit même la fin de l'histoire

- fin identifiable

- situation finale présente

- le texte est cohérent : il a une fin

Ces enseignants sont sensibles à la présentation matérielle du texte et à la saillance de la macroproposition correspondant à la situation finale.

Progression thématique

Si une majorité d'enseignants évalue explicitement la reprise des éléments de la fin du texte amorce au début du texte produit, quatorze d'entre eux se posent aussi la question de la dynamique de progression 
des énoncés au sein même du texte de Précilia. Ils interrogent tout particulièrement un changement de thème en fin de dialogue (- On s'en occupe ! dirent les spécialistes. - Pouvez-vous nous ramener ? direntils tous en chœur). Les annotations (incohérence ; quel rapport ?; pas de lien ; rupture dans la continuité du texte) posent selon nous deux questions: 1) la perception d'une rupture thématique comme une progression cohérente (Combettes, 1988) : nous observons un énoncé qui possède une valeur illocutoire particulière : en disant «on s'en occupe », les spécialistes modifient le monde. Cet énoncé déclaratif, rendant effective la résolution du problème, permet aux compagnons de changer de sujet et à l'élève scripteur de changer de thème : la dynamique d'actions se poursuit dans une résolution en plusieurs temps. 2) Le rapport thème / topic dans la construction du sens (Carter Thomas, 2000) : il semble que si une phrase ou une partie de texte ne se trouvent pas en rapport avec un topic construit à un moment donné de la lecture d'un texte, cette phrase ou cet extrait ne seront pas jugés cohérents. Les enseignants qui sanctionnent le tour de parole « Pouvez-vous nous ramener ? » semblent demander le développement du scénario « On s'en occupe. »

D'un point de vue théorique, le thème phrastique et le topic sont à distinguer, mais l'on voit que la perception d'un topic joue un rôle dans le traitement d'une suite d'énoncés formant un texte. Ainsi, les aspects linguistiques s'articulent aux notions pragmatiques et sémantiques.

Ce fait nous conduit à explorer plus précisément la manière dont les enseignants apprécient les informations véhiculées par le texte de Précilia.

\subsection{Effets de monde}

\subsubsection{A propos du requin évoqué dans le texte amorce}

Les remarques portent sur les propriétés sémantiques d'un lexème repris du texte source: quatre enseignants ne considèrent pas «le requin» (occurrence de la première phrase du texte de Précilia) comme une donnée morpho sémantique allant de soi : ils soulignent le déterminant anaphorique et notent :

- il n'est pas évident que l'ombre dans la mer soit forcément un requin

- découverte

- on ne savait pas encore qu'il s'agissait d'un requin

- on découvre ici que l'ombre est en réalité un requin mais son apparition est trop brutale. Le suspens n'est pas conservé.

Et plus surprenant :

- pas de cohérence avec le texte initial. L'élève n'a pas pris en compte que la silhouette appartenait à un homme et non à un requin

- incohérence : le nageur se transforme en requin

Ces annotations renvoient au rôle du contexte dans l'accès au sens : qu'est-ce qui est dit et/ou présupposé dans le texte amorce? L'isotopie générique dominante est celle du danger relatif aux requins. Mais les informations sont paradoxales : la lecture de «nous venions d'attraper des requins » implique l'idée que des individus sans matériel lourd et sans protection peuvent attraper des requins alors qu'à la fin de l'extrait, le requin devient un animal redoutable. Par ailleurs, les substituts de « requin » s'actualisent soit par une classe à grande généralité //une ombre//, qui par rétroaction se voit attribuer le trait /angoissant/, soit le SN 'le nageur', qui s'inscrit dans le cadre d'une classe //piscine// : 'une petite séance de natation ; notre bain; une rapide trempette'. Au final, le texte oppose deux domaines : le danger et le plaisir du bain, le second activant le trait /humain/. Par ailleurs, le substantif « nageur » est introduit par un indéfini (« un nageur »), ce qui peut impliquer une sélection thématique nouvelle fondée sur une inférence construite à partir de l'activation du trait /humain/. Les remarques de ces enseignants posent crucialement la question du parcours interprétatif et du lien entre progression thématique et saillance : le texte amorce, par sa construction, propose des mises en relief : au niveau phrastique, «nageur » est sans aucun doute un mot clef, introduit au moment de la complication. Mais au niveau textuel, la phrase clef qui accentue le 
sens global du texte est la première. Elles posent aussi la question de la perception de traits sémantiques récurrents : en quoi pour le lecteur ombre, requin, nageur forment-ils une unité sémantique?

\subsubsection{A propos des éléments de résolution}

S'appuyant sur des compétences encyclopédiques et intertextuelles apparemment partagées, une majorité d'enseignants questionne ou sanctionne la présence de certains objets sur le radeau et la plupart des actions des personnages, par exemple :

- le vocabulaire et le cadre du texte de départ ne sont pas repris sur un radeau une pelle ? un téléphone?

- le cadre du récit adopté par l'élève ne correspond pas aux moyens suggérés par l'extrait

- l'utilisation du téléphone semble peu probable

- les spécialistes arrivent d'on ne sait où

- $\quad$ spécialistes de quoi ?

- $\quad$ comment ont-ils pu être localisés dans cette immensité ?

- la résolution du texte est contradictoire avec l'histoire

- $\quad$ fin qui me paraît peu plausible

- où se trouvent les personnages ? Peuvent-ils rentrer chez eux si facilement? En ont-ils envie d'après toi ?

Il apparaît que le type de scénario situationnel proposé par l'élève montre plusieurs contradictions avec les éléments du texte amorce et tout particulièrement du paratexte. Il y a une sorte de conflit entre le monde retenu par les enseignants lors de la lecture du texte source et celui proposé par Précilia, soit d'un côté une expérience scientifique qui a pour enjeu de se défaire de toute modernité et de l'autre, l'intervention des moyens modernes et sophistiqués.

En mettant en perspective les annotations ci-dessus avec toutes les remarques ayant trait à la question de l'homogénéité sémantique du texte, nous nous interrogeons sur la tension qui s'exerce dans l'interprétation d'un texte entre l'identification des propriétés sémantiques des unités linguistiques et l'expérience globale des textes. En effet, les mêmes enseignants semblent " oublier » les compagnons de voyage de Knut mais gardent saillant le scénario « radeau perdu en pleine mer ». Ces deux éléments se trouvent au même endroit : dans le paratexte. Il semble ici que le sens se construit d'abord en fonction des contraintes exercées par un texte en tant que pratique inscrite dans un espace discursif déjà là : ce qui relève de la culture commune, du script construit culturellement, et qui reste saillant en mémoire, c'est l'association entre radeau et dénuement. Il semble qu'un genre de référence crée un effet d'univers sémantique (Rastier, 2007) et inhibe certaines informations du texte.

\subsection{Modes de lecture des enseignants}

Lorsque l'on compare le contenu des remarques des enseignants en cours de texte et en commentaire final, deux phénomènes deviennent évidents :

Alors que l'exercice de continuation textuelle est régulièrement choisi dans le cadre scolaire afin d'évaluer la compétence des élèves à gérer des règles de "répétition » à tous les niveaux (noms des personnages, référentiation, substitutions, inférences, etc.), les enseignants semblent pour une part assez importante, ne se préoccuper dans une première lecture que de la cohérence interne au texte produit. Les annotations dans ce cadre ne font pas référence au texte amorce (30\%). En revanche, 93\% des enseignants interrogent le lien entre texte amorce et texte produit dans le commentaire final.

Et certains enseignants semblent avoir un jugement évolutif du texte de Précilia : un même enseignant annote le texte de l'élève de façon négative (Knut absent ; téléphone $\rightarrow$ situation non maîtrisée ; difficulté de maîtrise des temps ; téléphone ? spécialistes ?) et conclut : suite réussie. Un autre écrit sur le texte : la liaison avec le texte de départ est correcte ; souligne ne voulurent plus aller en mer et note : Bien! A 
exploiter ! et porte le jugement suivant en commentaire : le dialogue est pauvre, les personnages ne sont pas exploités.

Sans porter nous même de jugement sur ces jugements, nous pouvons cependant nous poser la question du rapport entre niveau local et niveau global dans la construction de la cohérence et reprendre l'idée de double composante horizontale et verticale dans l'interprétation : d'un côté, il s'agit de juger, au fur et à mesure de la mise en mémoire d'une unité sémantique, en quoi elle est liée à la précédente (processus ascendant); de l'autre, il s'agit d'activer des scénarios mais aussi des représentations de pratiques langagières et de les confronter au texte produit (processus descendant).

\section{Bilan: la cohérence, une question de degrés dans l'exigence interprétative}

$\mathrm{Au}$ terme de notre analyse, nous pouvons constater que notre hypothèse de départ, soit l'existence d'un écart entre les définitions déclarées et les définitions implicites présentes dans les jugements est vérifiée. En effet, si les enseignants s'appuient sur des faits de langue pour juger le texte de Précilia, ils ne le font pas forcément sur les unités les plus attendues : il n’y a par exemple, aucun remarque concernant les indicateurs temporels quelques heures plus tard; après quelques heures : du point de vue de la cohérence du récit, représentent-ils les « bons » sauts dans le temps? De même, peu d'enseignants (6) interrogent la cohérence énonciative lors du passage du passé simple au présent de l'indicatif dans le récit, le repérage et l'emploi des temps du récit représentant pourtant le cœur des leçons de grammaire textuelle scolaire. Nous avons noté supra qu'un autre phénomène lié à la cohérence énonciative, soit la voix narrative, a très peu été jugé par les enseignants.

La cohérence thématique s'avère en revanche au centre des jugements de cohérence des enseignants : de ce point de vue, il semble qu'il existe des variations inter-individuelles en fonction des exigences et des modes de lecture des évaluateurs. Ainsi, par exemple, des avis sur la clôture du récit sont contradictoires à la fois du point de vue de l'intelligibilité et du «pouvoir d'intéressement» (Dumortier, 2005) (fin qui me paraît peu plausible versus récit qui a une fin et une belle fin! Happy end!). Nous avons relevé des différences de gloses sur la continuité thématique du début du texte de Précilia, qui montrent une tension entre cohésion interphrastique et inférences de liaison : pour accepter que le texte de l'élève commence par la série de prénoms, il faut faire l'effort de dépasser l'information présente en mémoire immédiate (ils atteignirent l'embarcation en même temps) et réaliser une association plus lointaine entre les compagnons évoqués dans le paratexte et les prénoms proposés par l'élève. Pour donner un dernier exemple, prenons un jugement «en creux »: si 14 enseignants considèrent la demande des compagnons insérée dans le dialogue comme une rupture (-pouvez-vous nous ramener?), 39 ne portent aucun jugement sur cette section du texte : l'on pourrait avancer l'hypothèse que l'ellipse temporelle n'est pas considérée comme fautive par des enseignants ayant estimé que l'élève, en employant un verbe performatif (on s'en occupe) concentre l'attention et l'intérêt du lecteur sur la suite de son récit, soit un point qui semble important pour elle : faire en sorte que ses héros retrouvent sérénité et sécurité.

\section{Conclusion : la cohérence, quels problèmes pour la didactique ?}

L'école est le lieu de l'apprentissage d'une certaine norme. Comment penser la question de la norme en matière de cohérence?

Si nous suivons notre analyse, nous devons admettre que la cohérence relève plus d'une imbrication de contraintes cognitives, pragmatiques, linguistiques, socio-langagières que de la norme. Il nous semble que l'étude de ces contraintes doive faire partie des objectifs de formation des enseignants.

Il nous faut en effet compléter la représentation que les enseignants ont de la cohérence : d'une part en développant leur connaissance de la portée des marques cohésives et de leur pouvoir instructionnel (et ainsi réfléchir aux éléments textuels qui impliquent plutôt une mise en sens locale et à ceux qui ont un effet plus large), d'autre part en leur donnant des outils d'analyse de leurs propres jugements. 
Les approches théoriques que nous avons sélectionnées nous semblent donc devoir être problématisées dans le cadre de la formation. Exercice certes très difficile ${ }^{11}$ mais qui permettra cependant une analyse plus fine par les enseignants des enjeux de la lecture - évaluation d'un texte d'élève : le jugement de cohérence, loin de se concentrer sur la liste des éléments morphosyntaxiques déclinés dans les programmes, s'appuie sur des aspects sémantiques et des procédures psychologiques que les enseignants mettent en œuvre de façon implicite. Un rôle de la formation au jugement de la cohérence de textes d'apprentis scripteurs pourrait être l'accompagnement à la conscientisation de la manière dont chacun gère les différents niveaux de contrainte textuelle.

\section{Références bibliographiques}

Adam, J.-M. (1999). Linguistique textuelle, des genres de discours aux textes. Paris : Nathan Université.

Adam, J.-M. (2002). Cohérence. Dictionnaire d'analyse du discours. Paris : Seuil.

Adam, J.-M. (2005). La linguistique textuelle, Introduction à l'analyse des discours. Paris : Armand Colin.

Boré, C. (2004). L'écriture scolaire : langue, norme, « style», quelques exemples dans le discours rapporté. LINX, $n^{\circ} 51$, p. 91-106.

Bronckart, J.-P. (1997). Activité langagière, textes et discours, Pour un interactionisme socio-discursif. LausanneParis : Delachaux et Niestlé.

Carter-Thomas, S. (2000). La cohérence textuelle. Paris : L'harmattan.

Charolles, M. (1995). Cohésion, cohérence et pertinence du discours. Travaux de linguistique, n²9, p.125-151.

Charolles, M. (2005). Cohérence, pertinence et intégration conceptuelle. Des discours aux textes modèles et analyses. P. Lane. (éd). Rouen : Publications des Universités de Rouen et du Havre, p. 39-74.

Charolles, M. (2006). De la cohérence à la cohésion du discours. Cohérence et discours. F. Calas (dir). Paris : Presses de l'Université de Paris-Sorbonne, p. 25-38.

Chuy, M., Rondelli, F. (à paraître). Traitement des contraintes linguistiques et cognitives dans la construction de la cohérence textuelle. Langages. Paris : Armand Colin.

Combettes, B., Tomassone R. (1988). Le texte informatif, aspects linguistiques. Bruxelles : De Boeck Université.

Coirier, P., Gaonac'h, D., Passerault, J.-M. (1996). Psycholinguistique textuelle, approche cognitive de la compréhension et de la production des textes. Paris : Armand Colin /Masson.

Eco, U. (1985). Lector in Fabula. Le rôle du lecteur. Paris : Grasset.

Gombert, J.-E. (1997). Ontogenèse de la machine à lire. Cahiers de Beaumont, $n^{\circ}$ 74-75, p. 27-34.

Grice, H. P. (1979). Logique et conversation. Communications, $n^{\circ} 30$, p.57-72.

Petitjean, A. (2007). Corpus et genres : quelles interactions ? Discours, diachronie, stylistique du français, Etudes en hommage à Bernard Combettes. O. Bertrand, M. Charolles, J. François, C. Schnedecker (éds). Berne : Peter Lang.

Plane, S. (2006). Singularités et constantes de la production d'écrit - L'écriture comme traitement de contraintes. DIPTYQUE, $n^{\circ} 5$, p. 33-54.

Rondelli, F. (2008). La cohérence textuelle : rapport à la langue, à soi, à l'autre ? Actes des Xèmes Rencontres Jeunes Chercheurs de l'école doctorale 268 Langages et Langues, 12 mai 2007. Paris : ILPGA.

Perruchet, P. (1989). Les automatismes cognitifs. Liège : Mardaga.

Rastier, F. (1987) Sémantique interprétative. Paris : Presses Universitaires de France.

Rastier, F. (2001). Arts et sciences du texte. Paris : Presses Universitaires de France. 
Rastier, F. (2007). Du réalisme au postulat référentiel. Revue Texto! [en ligne]téléchargeable au format PDF : http://www.revue-texto.net/Inedits/Rastier/Rastier_Postulat.pdf

Sperber, D. \&Wilson, D. (1989). La pertinence. Paris : Editions de Minuit.

${ }^{1}$ Ministère de l'Education nationale et de la Culture (1992), La maîtrise de la langue à l'école, Paris : Savoir Livre CNDP.

${ }^{2}$ Bulletin Officiel hors-série n5 du 12 avril 2007, page 79.

${ }^{3}$ Dans le cadre de notre thèse, nous analysons des définitions écrites de la cohérence proposées par des professeurs des écoles stagiaires. Le critère définitionnel choisi majoritairement est celui de « l'enchaînement logique des phrases », décliné en temps verbaux, ponctuation, connecteurs, anaphores.

${ }^{4}$ Charolles (2005) expose le fait, qu'il discute ensuite, que «tout le monde est aujourd'hui à peu près d'accord pour considérer que la cohérence est un principe général d'interprétation du discours qui s'applique [...] à toute séquence d'énoncés du moment que ceux-ci sont produits à la suite ».

${ }^{5}$ Ce qui montre la place croissante que prend le texte en linguistique ; voir Legallois 2006.

${ }^{6}$ Bronckart 1997.

${ }^{7}$ Voir, entre nombreux autres, le $n^{\circ} 36$ de la revue Pratiques sur le travail en projet, le $n^{\circ} 26-27$ de la revue Repères sur l'apprentissage de l'écriture à l'école élémentaire ou l'ouvrage de Claudine Garcia-Debanc, L'élève et la production d'écrits, collection Didactique des textes, Metz.

${ }^{8}$ Annexe 1.

${ }^{9}$ Annexe 1.

${ }^{10}$ Annexe 2.

${ }^{11}$ Voir sur la question l'article d’Elisabeth Nonnon dans Pratiques n97-98, juin 1998.

\section{Annexes}

\section{Annexe 1 : texte amorce}

\section{A bord du Kon Tiki}

Le Kon-Tiki est un radeau qui fut construit pour un jeune savant norvégien. Ce radeau, semblable à ceux des ancêtres des Indiens du Pérou, lui permit, avec cinq compagnons, de traverser l'Océan Pacifique, sans moteur et sans voiles, en se laissant entraîner par les courants.

Un jour, Knut eut sans le vouloir une petite séance de natation avec un requin. Il nous était interdit de nous éloigner pendant notre bain à cause de la déviation du radeau et à cause des requins. Mais un jour où la mer était particulièrement calme et où nous venions d'attraper les requins qui nous avaient suivis, une rapide trempette fut permise. Knut plongea le premier. Il s'était beaucoup éloigné avant de remonter à la surface pour revenir en nageant le crawl. A ce moment, nous vîmes une ombre plus grande que son corps monter derrière lui. Nous lançâmes un cri d'alarme, et il se dirigea de toutes ses forces vers le bord du radeau. Mais l'ombre appartenait à un nageur encore plus remarquable qui, s'élançant des profondeurs, gagnait sur Knut. Ils atteignirent l'embarcation en même temps.

\section{Annexe 2 : texte de Précilia}


ISBN 978-2-7598-0358-3, Paris, 2008, Institut de Linguistique Française

Didactique et enseignement, français langue maternelle, français langue seconde DOI $10.1051 / \mathrm{cmlf08139}$

Joël, Jean-Laurent, Josselin, Jimmy essayèrent d'accélérer mais le requin ne tombait pas. Jimmy prit une pelle et assomma le requin. Josselin prit le téléphone et appela des spécialistes. Quelques heures plus tard, les spécialistes arrivèrent et ils leur posent quelques questions.

- $\quad$ Où est le requin ? dit un spécialiste.

- $\quad$ Il est là ! crient les enfants en chœur.

- On s'en occupe ! dirent les autres spécialistes.

- $\quad$ Pouvez-vous nous ramener ? dirent -ils tous en chœur.

- Mais bien sûr!

Ils repartirent tous ensemble avec le requin et après quelques heures, ils entrèrent dans leur maison, contents de rentrer et ne voulurent plus aller en mer ! 